\title{
Quantitative assessment of structural and compositional colors induced by femtosecond laser: A case study on 301LN stainless steel surface
}

Xinying Shi ${ }^{\mathrm{a}, \mathrm{b}, *}$, Zhongjia Huang ${ }^{\mathrm{c}, *}$, Miku J. Laakso ${ }^{\mathrm{d}}$, Frank Niklaus ${ }^{\mathrm{d}}$, Rafal Sliz ${ }^{\mathrm{e}}$, Tapio

Fabritius ${ }^{\mathrm{e}}$, Mahesh Somanif ${ }^{\mathrm{f}}$, Tun Nyo ${ }^{\mathrm{f}}$, Xiao Wang ${ }^{\mathrm{g}}$, Meng Zhang ${ }^{\mathrm{g}}$, Gang Wang ${ }^{\mathrm{c}}$, Jukka

$\mathrm{Kömi}^{\mathrm{f}}$, Marko Huttula ${ }^{\mathrm{b}}$, Wei Cao ${ }^{\mathrm{b}, \mathrm{c}}$

a School of Physics and Electronic Engineering, Jiangsu Normal University, Xuzhou, 221116, China.

${ }^{\mathrm{b}}$ Nano and Molecular Systems Research Unit, University of Oulu, P.O. Box 3000, FIN90014, Oulu, Finland.

${ }^{\mathrm{c}}$ School of Mechanical and Automotive Engineering, Anhui Polytechnic University, Wuhu, 241000, China.

${ }^{\mathrm{d}}$ Department of Micro and Nanosystems, KTH Royal Institute of Technology, Stockholm, Sweden.

e Optoelectronics and Measurement Techniques Research Unit, University of Oulu, P.O. Box 4500, FIN-90014, Oulu, Finland.

${ }^{\mathrm{f}}$ Materials and Mechanical Engineering, Center for Advanced Steels Research, University of Oulu, P.O. Box 4200, 90014, Oulu, Finland.

g Department of Physics, East China University of Science and Technology, Shanghai 200237, China.

* Corresponding authors at School of Physics and Electronic Engineering, Jiangsu Normal University, Xuzhou, 221116, China and Nano and Molecular Systems Research Unit, University of Oulu, P.O. Box 3000, FIN-90014, Oulu, Finland (X. Shi); School of Mechanical and Automotive Engineering, Anhui Polytechnic University, Wuhu, 241000, China (Z. Huang).

E-mail addresses: xshi@jsnu.edu.cn (X. Shi), hzj@ahpu.edu.cn (Z. Huang). 


\begin{abstract}
The topic of durable coloration and passivation of metal surfaces using state-of-theart techniques has gained enormous attention and devotion with unremitting efforts of researchers worldwide. Although femtosecond laser marking has been performed on many metals, the related coloration mechanisms are mainly referred to structural colors produced by the interaction of visible light with periodic surface structures. Yet, general quantitative determination of the resulting colors and their origins remain elusive. In this work, we realized quantitative separations of structural colors and compositional pigmentary colors on 301LN austenitic stainless steel surfaces that were treated by femtosecond laser machining. The overall color information was extracted from surface reflectance, with structural color given by numerical simulations, and oxide compositions by chemical state analysis. It was shown that the laser-induced apparent colors of 301LN steel surfaces were combinations of structural and compositional colorations, with the former dominating the angular response and the latter setting up the brownish bases. In addition to the quantification of colors, the analysis method in this work may be useful for the generation and specification of tailored color palettes for practical coloration on metal surfaces by femtosecond laser marking.
\end{abstract}

Keywords: Laser coloration, Structural color, Compositional color, Femtosecond laser marking 


\section{Introduction}

Pulsed lasers have been widely used to produce structural colors on metallic surfaces $^{1,2}$. Periodic micro and nanostructures can be formed on metallic surfaces by the interference of femtosecond, picosecond or nanosecond laser pulses with the excited surface plasmon polaritons ${ }^{3,4}$ and the surface structures can be precisely controlled ${ }^{5,6}$. Such laser-induced periodic surface structures (LIPSSs) are attributed as the main origin of apparent colors resulting from the laser coloration. It is worth mentioning that LIPSSs are also employed in medical applications to improve biocompatibility by controlling cell adhesion and migration ${ }^{7,8}$ and in surface chemistry applications to increase wettability by fabricating the spacings and patterns ${ }^{9,10}$. LIPSSs involve fundamental challenges due to complicated inter- and intra-pulse light-matter interactions, and technical advances in practical applications thanks to their straightforward laser-induced processes. Thus, LIPSSs remain "evergreen" in both materials science and engineering ${ }^{11}$. The LIPSSs affect the coloration by diffraction grating effects ${ }^{1}$ and also plasmonic effects if there are metallic nanoparticles decorating the LIPSSs ${ }^{12}$. Complete color palettes have since been realized by laser irradiation on both noble-metal and stainless-steel surfaces ${ }^{12,13}$. Recently, a mechanoresponsive color change was realized by varying the LIPSS spacings, and such surface features can be replicated by polymer casting ${ }^{14}$.

Despite these recent advances, the elusive quantification of laser-induced colors hinders broad implementation of laser coloration in practical application. Besides forging surface structures, the exposure of metal alloy surfaces by fast and intensive laser pulses will also induce rearrangement of surface chemical states ${ }^{15}$ or even phase transformations ${ }^{16}$. The laser-induced chemical compositions (metal oxides, spinels, etc.) have their intrinsic 
pigmentary colors. As a result, such compositional pigmentary colors also contribute to the coloration $^{17,18}$. This, however, complicates the dominant role of LIPSSs in metal surface colorations. Thus, a reliable quantitative description of structural and compositional colors formed during laser treatment is a critical requirement for bringing laser coloration towards practical applications.

Quantifying compositional colors requires careful determination of surface chemical species and their contents after laser treatments. For example, stainless steels can be polished to obtain extremely flat surfaces that are well suited for subsequent laser-induced coloration. During the formation of LIPSSs, the laser treatment may also cause metal oxidation and metal alloy formation in the steel surface. Besides colorization of the steel surfaces, these effects can also lead to improved corrosion resistance and hydrophobicity of the treated surfaces ${ }^{19}$. Among various stainless steel grades, AISI 301LN is an austenitic stainless steel grade having a good balance of properties in respect of high strength, ductility and strain hardening capacity, besides excellent corrosion resistance ${ }^{20,21}$. This in turn enables extensive forming without necking feasible and commonly finds applications as automotive components, rail car structural components, airframe sections, etc. Considering the environmental issues, it is interesting to render metal surfaces with different colors without coating by conventional chemical or electrochemical technique ${ }^{22}$.

In this paper, we present a quantitative study of femtosecond laser coloration of 301LN stainless steel surfaces and the prevalent structural and compositional mechanisms involved in the color formation. We have characterized the reflectance behavior of the laser-treated surfaces through ellipsometry and spectrophotometry measurements, and we also have computed the reflectance by finite differential time domain (FDTD) methods. 
We found that the simulated and experimental reflectance spectra match very well for the main peaks, thus leaving differences in relatively limited values. We discussed the agreements and discrepancies in respect of individual contribution of the structural colors and compositional pigmentary colors. The compositional coloration was further confirmed through surface chemical analysis. Our quantitative analysis of the femtosecond laserinduced coloration of 301LN stainless steel surfaces confirms that the color formation is due to a combination of structural and compositional coloration.

\section{Experimental section}

\subsection{Femtosecond laser processing}

Femtosecond laser coloration was applied on mechanically-polished stainless steel (AISI 301LN) substrates. In the coloration experiments, we used a femtosecond laser (Spirit 1040-4-SHG, Spectra-Physics) with a wavelength of $521 \mathrm{~nm}$, the pulse length of 293 fs (FWHM), and linearly polarized laser light. Laser pulses with the energy of $0.64 \mu \mathrm{J}$ were focused on a sample surface through an objective with a numerical aperture of 0.10 . By measuring the crater diameters induced by different laser fluences ${ }^{23}$, we found that the focused beam had the $1 / \mathrm{e}^{2}$ Gaussian beam radius of $8 \mu \mathrm{m}$, and a damage threshold energy of $0.1 \mu \mathrm{J}$ for a single pulse on steel surface. From these values we calculated the damage threshold peak fluency of $0.09 \mathrm{~J} / \mathrm{cm}^{2}$ for a single laser pulse. This threshold fluency is comparable to a value reported for stainless $\operatorname{steel}^{24}$.

The stainless steel samples were colorized by moving them laterally underneath the laser focus, thus placing individual laser pulses over the sample surface with an even pulse pitch. The pulse pitch was varied between the samples with the aim of obtaining different stable, i.e. non-iridescent, colors while at the same time keeping iridescence around one of 
the tilting axes. Each pulse pitch corresponds to an average fluency over the sample surface because the pulse energy was kept constant. An average fluency of $1.6 \mathrm{~J} / \mathrm{cm}^{2}(6.4 \mu \mathrm{m}$ pulse pitch) resulted to a blue stable color (Sample A) and an average fluency of $3.2 \mathrm{~J} / \mathrm{cm}^{2}(4.5$ $\mu \mathrm{m}$ pulse pitch) resulted to a light-green stable color (Sample B). The applied average fluencies ranged from 17 to 144 times the damage-threshold for a single-pulse peak fluency. The pulse pitches are comparable to the beam radius of $8 \mu \mathrm{m}$, indicating that only a small number of laser pulses affected any single point on a sample surface. This limited overlap between the pulses is compatible with the fact that the pulse energy used in the experiments $(0.64 \mu \mathrm{J})$ exceeds the damage-threshold energy for a single laser pulse $(0.1 \mu \mathrm{J})$. The pulse repetition rate was $625 \mathrm{~Hz}$ for Sample A and $885 \mathrm{~Hz}$ for Sample B, both values sufficiently low to avoid significant heat accumulation in the sample surfaces during laser processing.

\subsection{Characterization}

A scanning electron microscope (SEM, Zeiss Sigma) equipped with an energydispersive X-ray spectroscopy (EDAX Apollo X) analyzer was employed to study the LIPSSs on the 301LN surfaces. The heights of LIPSS layers were measured by a Bruker ContourGT-K surface profiler, and the profilograms were shown in Fig. S1 (Supplementary material). This technique is non-destructive and suitable for studying laser-induced structures on steel surfaces ${ }^{18}$.

In order to record and present the apparent colors of the laser-irradiated steel surfaces, the optical photographs of the samples surfaces were taken in two ways: (a) photos taken using a digital camera with natural light illumination and (b) photos taken by an optical microscope (Nikon Eclipse LV100DA-U). The color information was read from the photos 
and recorded in the RGB color model. The average RGB values of the samples were calculated by ImageJ software with the Color Histogram plugin ${ }^{25}$.

The optical properties of the laser-irradiated surfaces were measured with an ellipsometer (UVISEL-VASE Horiba Jobin-Yvon) and an Automated Spectroradiometric Measurement System (Optronic Laboratories). The angles of incidence were $70^{\circ}$ and $13^{\circ}$ for ellipsometry and spectrophotometry measurements, respectively. The refractory indices of the laser-irradiated surfaces determined through ellipsometry were the basic parameters used in the FDTD simulation.

The quantitative compositional study of the laser-treated surfaces was performed by X-ray photoelectron spectroscopy (XPS, Thermo Fisher Scientific ESCALAB 250Xi XPS System with $\mathrm{Al} \mathrm{K} \alpha \mathrm{X}$-ray source). The chemical states and relative contents of the elements $\mathrm{Fe}, \mathrm{Cr}, \mathrm{Ni}$ and Mn were characterized. The XPS spectra were calibrated with the reference value of adventitious $\mathrm{C} 1 s$ at $284.8 \mathrm{eV}$.

\subsection{Simulation}

Optical simulation work was performed by using the Lumerical FDTD Solutions software. The calculation was carried out based on the space and time partial derivatives of discrete time-dependent Maxwell's equations. Simulation models were established for the LIPSSs structures with and without metal nanoparticles. The refractive indices employed in each model were consistent with the data obtained from the ellipsometry measurements. More details of the simulation method can be found in previous studies of patterned nanostructure surfaces ${ }^{26,27}$. 


\section{Results and discussion}

\subsection{Surface morphologies and resulting colors}

Due to the ablation effect of the femtosecond laser pulses, the stainless-steel surfaces were reconstructed with micro and nano-scale structures. The resulting LIPSS morphologies of the two samples are shown in Fig. 1a and b. Created by the lasers using different fluencies $\left(6.25 \mathrm{~J} / \mathrm{cm}^{2}\right.$ for Sample A and $13.22 \mathrm{~J} / \mathrm{cm}^{2}$ for Sample B), the two samples are rendered with LIPSSs of different sizes. The surface of Sample A is covered by $280 \mathrm{~nm}$ wide ripples with a periodic repetition width of $420 \mathrm{~nm}$. Sample B has a relatively smaller surface structure with $230 \mathrm{~nm}$ wide ripples and a periodic repetition width of $380 \mathrm{~nm}$, in accordance with the higher average fluency employed ${ }^{28}$. The dimensions and periodicity of these structures are in the same scale as the wavelength of visible light, and thus interference with visible light should be expected, leading to iridescent structural colors. In addition, there are metallic nanoparticles distributed on the surfaces of both samples. These nanoparticles are mainly 20 - $50 \mathrm{~nm}$ in size (Fig. S2), with similar chemical compositions to the LIPSSs (Fig. S3). Apart from the LIPSSs, the nanoparticles on the surfaces usually create angle-independent (non-iridescent) colors via plasmonic effects ${ }^{12}$. The morphology of the sample surfaces is conceptually illustrated in Fig. 1c. The heights of the LIPSS topographies were determined by surface profile measurements (Fig. 1d). The above information shows that the two samples are covered by LIPSSs of different sizes in both the lateral and vertical dimensions. 

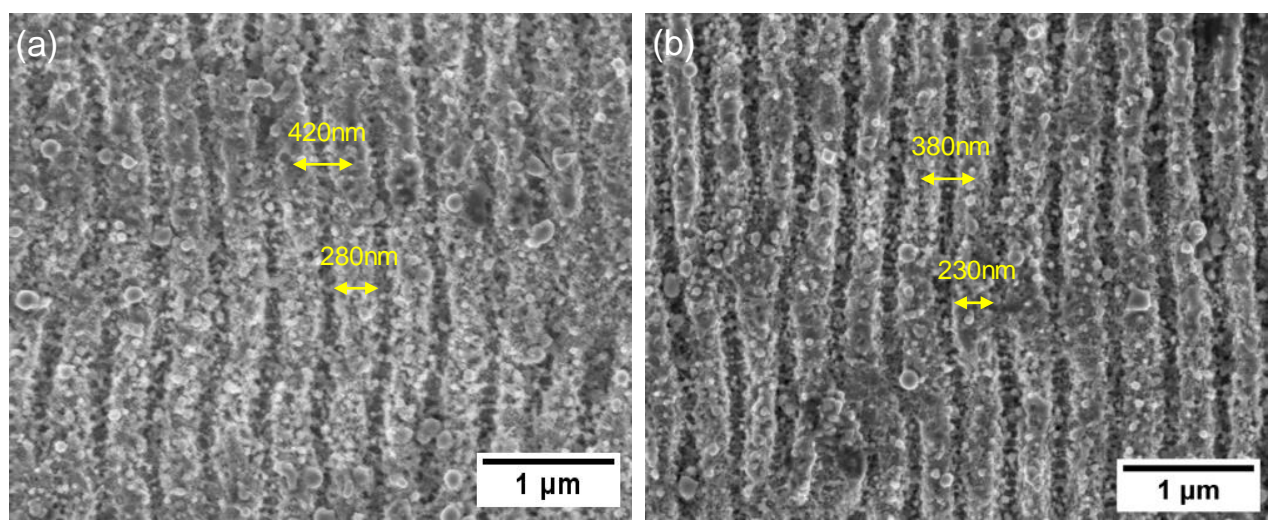

(c)
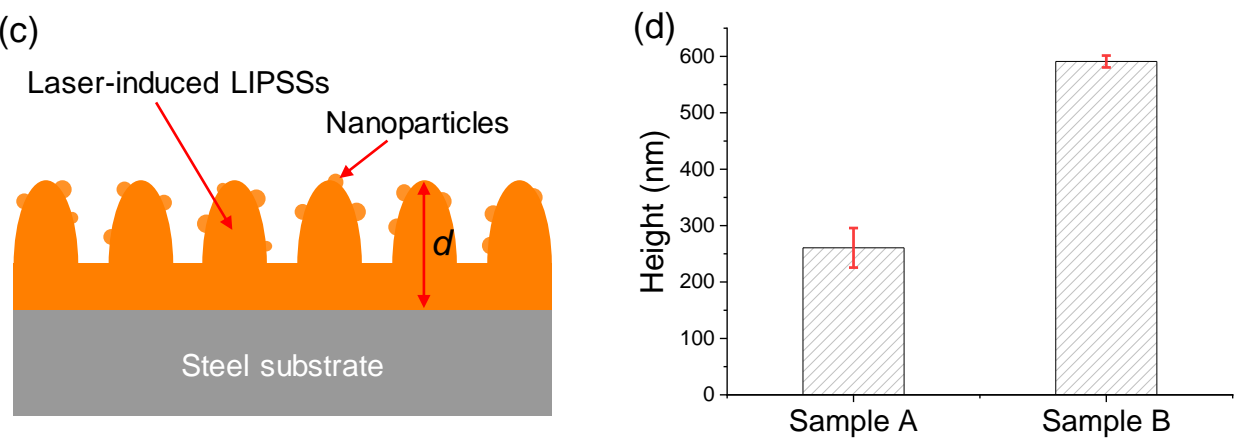

Fig. 1. The morphologies and thicknesses of the LIPSSs. (a) SEM image of Sample A. (b)

SEM image of Sample B. (c) Schematic of the morphology of the LIPSSs with randomly distributed nanoparticles. (d) Measured heights of the LIPSS topography (labelled as $d$ in panel c).

The laser-irradiated steel surfaces present iridescent colors which are typical features of structural coloration. Observed from different directions, the surfaces appear shiny and feature changeable colors. Fig. 2a-e show the appearance of the two sample surfaces at incident angles of $13^{\circ}$ and $70^{\circ}$, respectively. Such angle-dependent colors suggest that the LIPSSs dominate the coloration in comparison with the surface nanoparticles. 


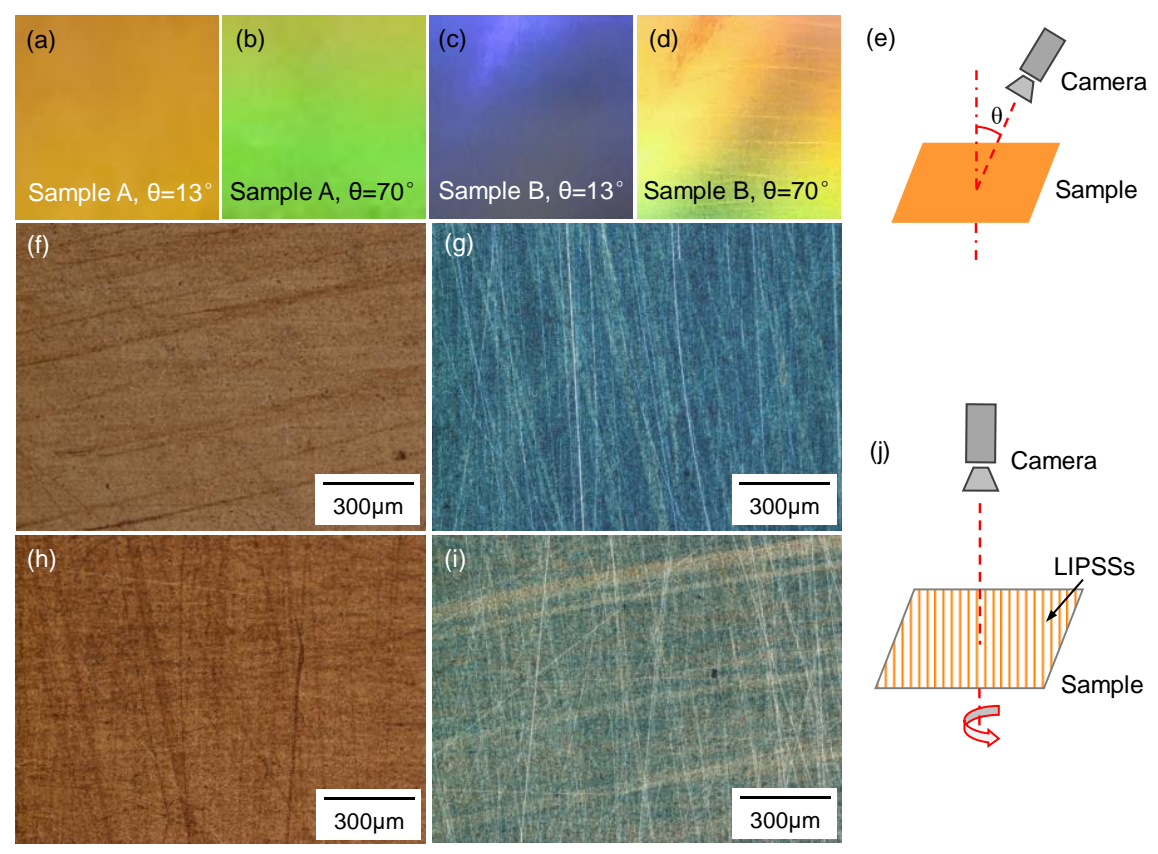

Fig. 2. (a-d) Photographs of the samples illuminated by natural light. (e) Schematic diagram illustrating the image acquisition from different directions. $\theta$ is the observation angle. (f-g) Optical microscopy photos of Sample A with the LIPSS trenches running parallel and perpendicular to the light polarization, respectively. (h-i) Photos of Sample B with the LIPSS trenches running parallel and perpendicular to the light polarization, respectively. (j) Schematic diagram of the optical microscopy measurements.

In order to quantitatively describe the induced colors, the RGB color model was employed for color calculation and presentation ${ }^{29}$. The RGB model presents colors in red, green and blue components. Each of the color components varies from 0 to 255 in accord with the brightness and color saturation. For each of the photographs in Fig. 2, the RGB values of each pixel is recorded and averaged to obtain the average color. The average colors are listed in Table S1 in the Supplementary material. It is worth noting that the light source employed to record the colors of Fig. 2a-d was natural light while the photos of Fig. 2f-i were taken with polarized light illumination. 
LIPSSs are typically sensitive to the polarizations of incident light. To demonstrate the influence of polarized light, we took photos of the samples with the camera kept over the sample surfaces (see Fig. 2f-i). The samples were illuminated by linearly polarized light and positioned with their LIPSS trenches parallel or perpendicular to the polarization of the illumination (Fig. 2j). In these two cases, the samples feature distinct colors. With a $90^{\circ}$ rotation along the normal line of the sample surfaces, both samples appeared from brownish to bluish in color. It is well known that light-matter interference is not effective when the polarization of the light is running in parallel to the grating alignment. In this aspect, the colors in Fig. $2 \mathrm{f}$ and $\mathrm{h}$ are less impacted by structural colors and closer to the intrinsic colors resulting from the surface compositions. Thus, the femtosecond laserpatterned surfaces feature tunable colors by altering either the incident angle or the polarization of the illuminating light.

\subsection{Structural coloration}

In accordance with the photographs in Fig. 2a-d, the reflectance was measured at two incident angles: $70^{\circ}$ by ellipsometry (Fig. 3a) and $13^{\circ}$ by spectrophotometry (Fig. 3b), respectively. The results show that the reflected light from the two incident angles leads to different reflectance spectra, which is the mechanism of structural colors. It is worth mentioning that in ellipsometry the reflectance is not measured directly. Instead, the complex refractive index, ellipsometric angles and other optical parameters are measured. With these measured data, the reflectance can be extracted based on a suitable model of the surface topography, including appropriate materials and dimensions ${ }^{30}$.

Sample A shows two reflectance peaks in the visible range at wavelengths of $384 \mathrm{~nm}$ and $601 \mathrm{~nm}$ while there is only one strong peak at $625 \mathrm{~nm}$ for the sample B as depicted in 
Fig. 3a. Both curves show high reflectance at long-wavelengths which in turn represent colors with high red and green contents. In the case of a $13^{\circ}$ incident angle (Fig. 3b), both reflectance spectra increase along with the wavelength, which is in agreements with previously published research results ${ }^{31}$. No obvious reflectance peaks were found in the measured wavelength interval in this case.
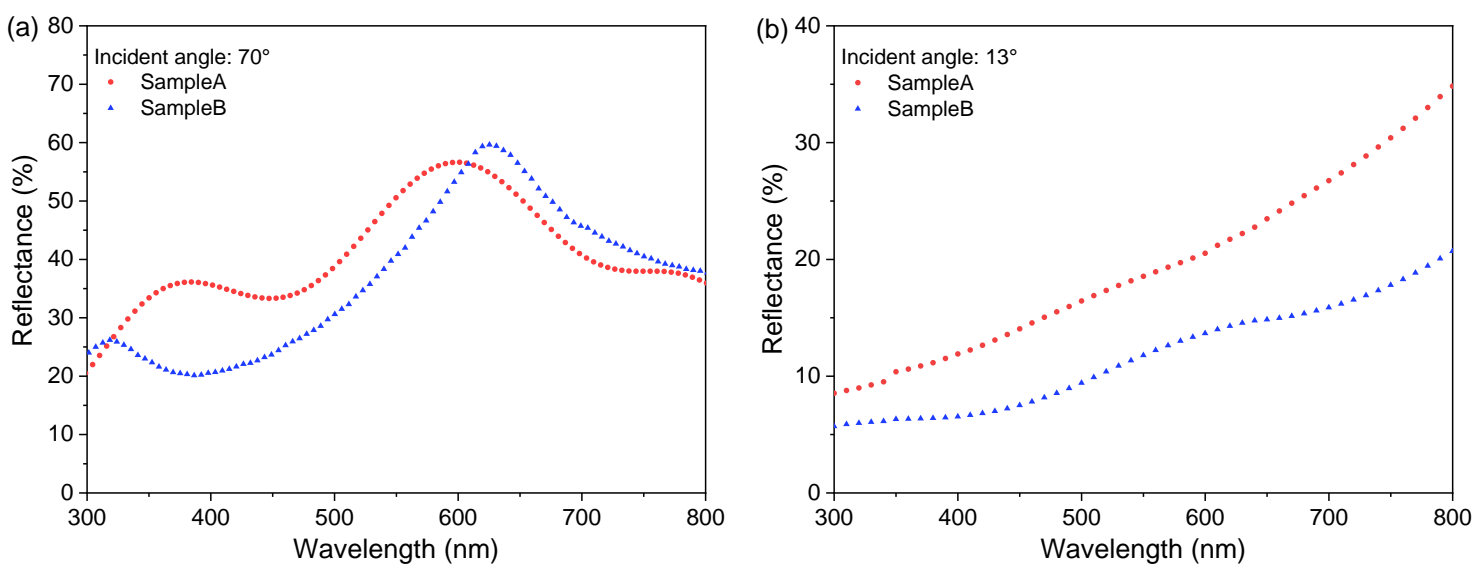

Fig. 3. Experimental reflectance spectra. (a) Reflectance results at an incident angle of $70^{\circ}$. (b) Reflectance at an incident angle of $13^{\circ}$.

To identify the influence of surface structures on the resulting colors, FDTD simulations were performed. The simulation was based on a model of the LIPSSs without nanoparticles (Fig. 4a) and a model of the LIPSSs with nanoparticles (NPs) on the surface (Fig. 4b), respectively. The simulation results (Fig. 4c,d) represent and predict the structural coloration effect. For Sample A (Fig. 4c), the simulated results based on the two models are almost identical, suggesting that the nanoparticles have little influence on the reflectance. This is reasonable because the nanoparticles are usually not related to iridescent colors ${ }^{12}$. Although the size, shape and composition of the nanoparticles contribute to the coloration ${ }^{32}$, the influence on the reflectance is limited and it is difficult to identify the spectra induced by the nanoparticles from the measured reflectance results. 
The reflectance spectra are dominated by the LIPSSs. The simulated reflectance curves matched the experimental reflectance quite well below a wavelength of $400 \mathrm{~nm}$ and near the region of the reflectance peak at $600 \mathrm{~nm}$, leaving obvious discrepancies in the wavelength range of $400-500 \mathrm{~nm}$. These discrepancies between the simulated and measured reflectance are located in the blue color region. In Fig. 4d, the simulation based on the model involving nanoparticles predicts more oscillations in the reflectance curve, but the simulated spectra based on the two models still followed similar trends. The differences between the experimental and simulated results are mainly in the wavelength intervals of $450-550 \mathrm{~nm}$ and $650-750 \mathrm{~nm}$, which are essentially responsible for green and red colors. Such discrepancies in reflectance raise questions towards the influence of intrinsic colors of surface chemicals on the overall color appearance.

(a)
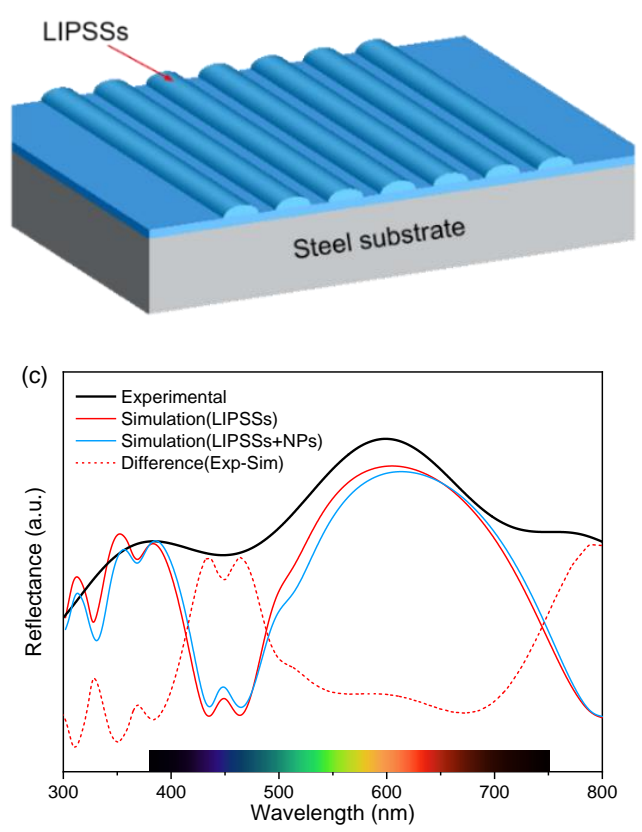

(b)
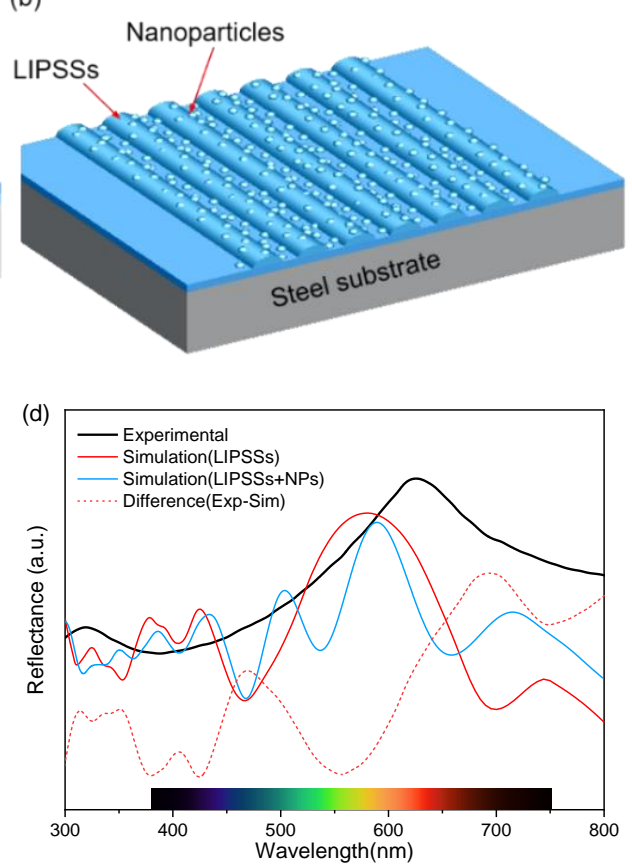

Fig. 4. Simulation results of the reflectance. (a) LIPSSs model. (b) LIPSSs+NPs model. (c) Measured and simulated reflectance of Sample A. (d) Measured and simulated reflectance of Sample B. The simulation results based on LIPSSs models and LIPSSs+NPs models are 
illustrated with red and blue curves, respectively. The dashed curves in red represent the difference between the experimental and the simulated reflectance. The spectral colors in the visible range $(380-750 \mathrm{~nm})$ are shown at the bottom of the graphs.

\subsection{Compositional coloration}

To elucidate the experimental and simulated reflectance, we examined the intrinsic colors of the chemical compositions in steel surfaces. Steel surfaces were reconstructed after laser irradiation, leaving metal oxides and spinels randomly arranged on the surfaces ${ }^{18}$. The metal oxides and spinels contribute to the surface coloration with their intrinsic colors. Such compositional coloration is ubiquitous for laser-treated metallic surfaces. In this work, we quantitatively studied the surface composition by XPS spectra and calculated the compositional distributions. The XPS spectra of $\mathrm{Fe} 2 p$, $\mathrm{Cr} 2 p$, Ni $2 p$ and $\mathrm{Mn} 2 p$ of samples A and B are shown in Fig. 5, the survey scans are presented in Fig. S4 and the peak fitting details are listed in Table S2. All spectra were fitted to identify specific chemical species. It can be seen in Fig. 5a that the steel surface was mostly oxidized to the spinel at $710.5 \mathrm{eV}$ and $\mathrm{Fe}_{2} \mathrm{O}_{3}$ at $712.5 \mathrm{eV}^{33}$, with very small amounts of $\mathrm{Fe}$ remaining on the surface. The spinel usually forms on steel surfaces during laser processing ${ }^{34}$ and has a typical form of $\mathrm{XY}_{2} \mathrm{O}_{4}(\mathrm{X}$ and $\mathrm{Y}$ are metallic ions with +2 and +3 valences, respectively) and can be

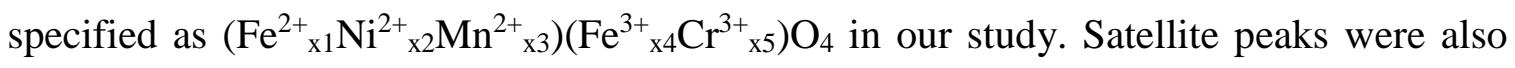
identified in the XPS spectra, which are typical for transitional metal elements. Metal oxides and the spinel structure were also identified in the $\mathrm{Cr} 2 p$, Ni $2 p$ and Mn $2 p$ spectra $^{35,36}$, as shown in Fig. 5b-d. 

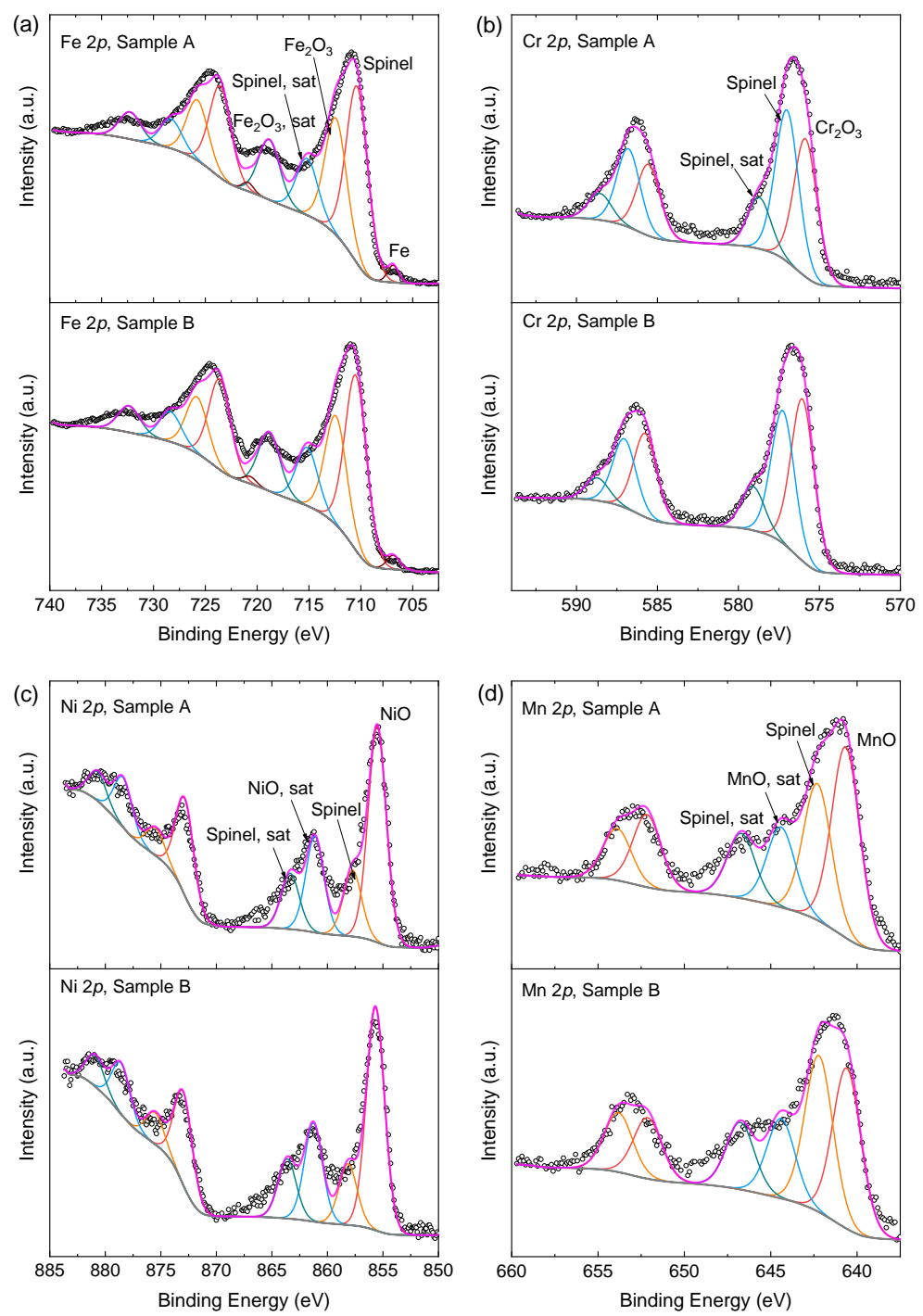

Fig. 5. XPS spectra of the laser-irradiated steel surfaces. (a) Fe $2 p$, (b) $\mathrm{Cr} 2 p$, (c) Ni $2 p$, (d) Mn $2 p$. The measured data, backgrounds, and fitting envelopes are illustrated with scatters, grey and magenta lines, respectively. The doublet peaks are plotted with the same color.

The fitted XPS results quantified the relative contents of the spinels in the two samples, which were determined as $\left(\mathrm{Fe}^{2+}{ }_{0.66} \mathrm{Ni}^{2+}{ }_{0.14} \mathrm{Mn}^{2+}{ }_{0.20}\right)\left(\mathrm{Fe}^{3+}{ }_{1.46} \mathrm{Cr}^{3+}{ }_{0.54}\right) \mathrm{O}_{4}$ and $\left(\mathrm{Fe}^{2+}{ }_{0.67} \mathrm{Ni}^{2+}{ }_{0.11} \mathrm{Mn}^{2+}{ }_{0.22}\right)\left(\mathrm{Fe}^{3+}{ }_{1.59} \mathrm{Cr}^{3+}{ }_{0.40}\right) \mathrm{O}_{4}$ for samples $\mathrm{A}$ and $\mathrm{B}$, respectively. The relative amount of metal oxides and spinel can be also calculated from the XPS data, as shown in Table 1. The RGB values of metal oxides were referred to the reports of pure minerals 
which could be referenced from previous reports ${ }^{37}$, while the spinel color was determined according to the relative amounts of the metallic ions. The main differences of the two spinels are the contents of $\mathrm{Fe}^{3+}$ and $\mathrm{Cr}^{3+}$. More $\mathrm{Fe}^{3+}$ ions render the spinel dark brown while less $\mathrm{Fe}^{3+}$ ions turn the spinel greenish or bluish ${ }^{38}$. On the other hand, $\mathrm{Cr}^{3+}$ ions in the spinel produce reddish colors ${ }^{39}$. Thus, the spinel colors of the two samples are specified as $(105$, $70,225)$ and $(210,90,165)$, respectively. The contribution of Fe was neglected due to the low contents in both samples. Based on a previous study, the chemical species contribute quantitatively to the apparent colors by their relative contens ${ }^{18}$. Compositional color of each sample can be computed by the product of its color matrix and content matrix via

$$
\left(\begin{array}{l}
R \\
G \\
B
\end{array}\right)=\left(\begin{array}{ccccc}
R_{1} & R_{2} & R_{3} & R_{4} & R_{5} \\
G_{1} & G_{2} & G_{3} & G_{4} & G_{5} \\
B_{1} & B_{2} & B_{3} & B_{4} & B_{5}
\end{array}\right)\left(\begin{array}{l}
C_{1} \\
C_{2} \\
C_{3} \\
C_{4} \\
C_{5}
\end{array}\right)
$$

where $R_{\mathrm{i}}, G_{\mathrm{i}}$, and $B_{\mathrm{i}}$ represent the red, green and blue intensity of the metal oxides and the spinel, respectively; $C_{\mathrm{i}}$ is the relative content of each chemical species. The calculated compositional colors are $(115,105,134)$ and $(145,112,118)$ for the Samples A and B, respectively.

Table 1. The relative content of metal oxides and spinels. The intrinsic RGB colors of the oxides and the specified RGB values of the spinels are also listed in the table. The cells are shaded with the RGB values therein.

\begin{tabular}{|c|c|c|c|c|c|c|c|}
\hline \multirow{2}{*}{ Sample } & \multirow{2}{*}{$\begin{array}{c}\mathrm{Fe}_{2} \mathrm{O}_{3} \\
(130,110,90)\end{array}$} & \multirow{2}{*}{$\begin{array}{c}\mathrm{Cr}_{2} \mathrm{O}_{3} \\
(80,121,95)\end{array}$} & \multirow{2}{*}{$\begin{array}{c}\mathrm{NiO} \\
(160,210,195)\end{array}$} & \multirow{2}{*}{$\begin{array}{c}\mathrm{MnO} \\
(105,110,85)\end{array}$} & \multicolumn{2}{|c|}{ Spinel } & \multirow{2}{*}{$\begin{array}{l}\text { Compositional } \\
\text { Colors }\end{array}$} \\
\hline & & & & & A & B & \\
\hline A & $44.90 \%$ & $10.20 \%$ & $7.38 \%$ & $7.47 \%$ & \multicolumn{2}{|c|}{$27.87 \%$} & $(115,105,134)$ \\
\hline B & $44.93 \%$ & $10.31 \%$ & $8.43 \%$ & $6.46 \%$ & \multicolumn{2}{|c|}{$27.62 \%$} & $(145,112,118)$ \\
\hline
\end{tabular}


From the data it can be seen that the two samples have slightly different chemical compositions, likely caused by the varied average laser fluency which was employed to create the surface structures. The compositional pigmentary colors thus can be calculated in accordance with the relative content of the oxides and spinels. As shown in Table 1, the compositional colors of the two samples are $(115,105,134)$ and $(145,112,118)$, respectively. The green and blue components of these colors are close to those shown in Fig. $2 \mathrm{~g}(66,112,131)$ and Fig. $2 \mathrm{i}(114,132,119)$, while they are very different from those shown in Fig. 2f $(134,97,62)$ and Fig. $2 \mathrm{~h}(136,84,44)$. Thus, the contribution of the structural colorations on the overall coloration is lessened due to specific optical geometry and light polarization.

A reflectance spectrum and an RGB triplet can be derived from one another by various computational algorithms 40,41 . Here we calculate the reflectance curves from the compositional RGB triplets by employing an iterative-least-log-slope-squared (ILLSS) algorithm $^{41}$. This algorithm generates reflectance spectra in the visible range (380 - 730 $\mathrm{nm}$ ) with a step of $10 \mathrm{~nm}$. Based on this algorithm, the compositional RGB values of the samples (Table 1) can be converted to the reflectance curves (Fig. 6). For both the samples, the converted reflectance curves roughly matched the main peaks of the difference between experimental and simulated reflectance spectra. Therefore, the experimental reflectance can be expressed by the combined contribution from the structural colors and compositional pigmentary colors. From Fig.4 and Fig. 6, it can be seen that the apparent colors are primarily determined by the structural colors because the simulated reflectance match the main features of the experimental results, while the surface chemicals also influence the apparent colors. 

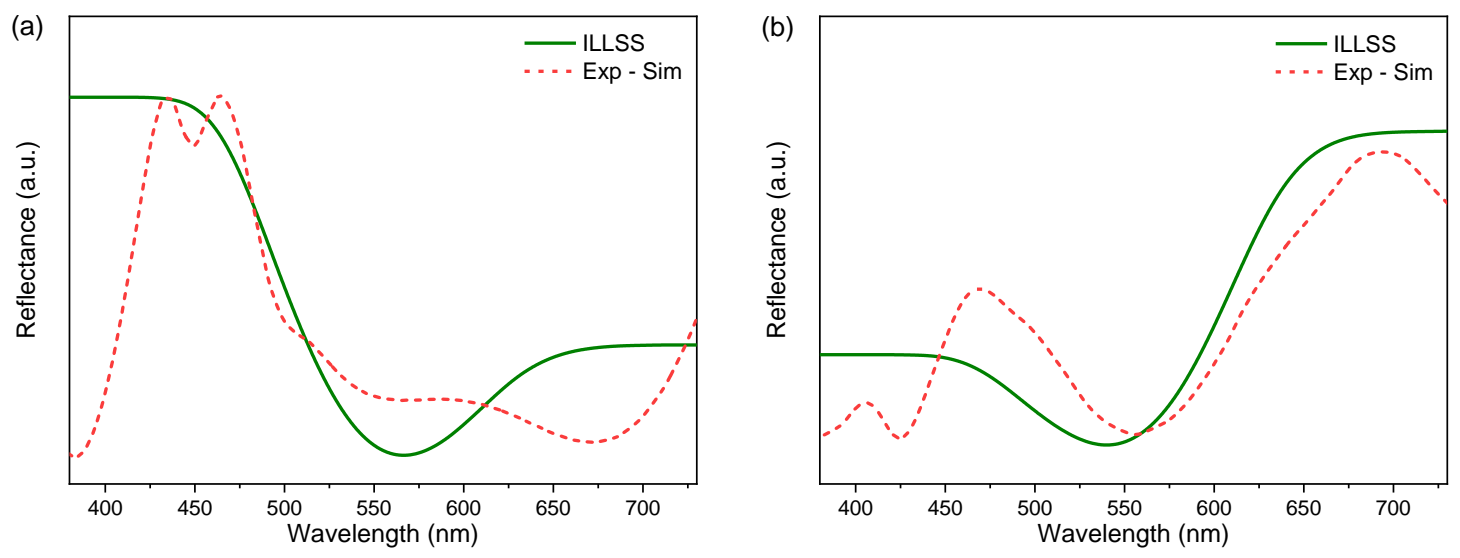

Fig. 6. Converted reflectance spectra from compositional RGB colors. (a) Sample A. (b)

Sample B. The green curves represent the reflectances converted from the compositional RGB values by the ILLSS algorithm. The red dashed curves represent the differences between measured and simulated reflectances.

\section{Conclusions}

In summary, we have quantitatively assessed the colorations and their origins on femtosecond laser-treated 301LN stainless steel surfaces. The processed steel surfaces demonstrated iridescent structural colors. Through experimental and simulated study of the reflectance properties, the laser-induced iridescence was found to origin mainly from the interaction between surface LIPSSs and visible light. However, discrepancies between measured and computed reflectance results remained obvious, and were further identified as the contributions of intrinsic colors from laser-induced oxides. Therefore, the femtosecond laser coloration is quantitatively defined by the combination of obvious structural colors and intrinsic pigmentary colors. Such a mechanism can also be generalized in various occasions, e.g., coloration on other metal or alloy surfaces by employing lasers of different pulses. In addition to colorizing the steel surfaces, the established routes and 
analysis offer a comprehensive understanding of laser coloration phenomena, which is expected to facilitate practical pulsed laser colorations.

\section{Acknowledgements}

The authors acknowledge the financial supports from the Academy of Finland (No. 311934, No. 296890), National Natural Science Foundation of China (No. 51704001), Natural Science and Technology Major Project of Anhui Province (No. 18030901035) and the Swedish Foundation for Strategic Research (SSF) (GMT14-0071). W.C. thanks the Anhui Provincial Grant for high-level platform construction. The Center of Microscopy and Nanotechnology of the University of Oulu is gratefully thanked for sample characterizations. Dr. Hui Zhang at Soochow University is acknowledged for assisting in the numerical simulation.

\section{Appendix A. Supplementary material}

Supplementary data to this article can be found online.

\section{References}

${ }^{1}$ Y. Yang, Y. Pan, P. Guo, Structural coloration of metallic surfaces with micro/nanostructures induced by elliptical vibration texturing, Appl. Surf. Sci. 402 (2017) 400-409

${ }^{2}$ A. Y. Vorobyev, C. Guo, Colorizing metals with femtosecond laser pulses, Appl. Phys. Lett. 92 (2008) 041914

${ }^{3}$ M. S. Ahsan, F. Ahmed, Y. G. Kim, M. S. Lee, M. B. Jun, Colorizing stainless steel surface by femtosecond laser induced micro/nano-structures, Appl. Surf. Sci. 257 (2011) 7771-7777

${ }^{4}$ A. Y. Vorobyev, C. Guo, Direct femtosecond laser surface nano/microstructuring and its applications, Laser Photonics Rev. 7 (2013) 385-407

${ }^{5}$ B. Dusser, Z. Sagan, H. Soder, N. Faure, J.P. Colombier, M. Jourlin, E. Audouard, Controlled nanostructures formation by ultra fast laser pulses for color marking, Opt. Express 18 (2010) 2913-2924

${ }^{6}$ E. I. Ageev, V. P. Veiko, E. A. Vlasova, Y. Y. Karlagina, A. Krivonosov, M. K. Moskvin, G. V. Odintsova, V. E. Pshenichnov, V.V. Romanov, and R. M. Yatsuk, Controlled 
nanostructures formation on stainless steel by short laser pulses for products protection against falsification, Opt. Express 26 (2018) 312530

${ }^{7}$ M. Martínez-Calderon, M. Manso-Silván, A. Rodríguez, M. Gómez-Aranzadi, J. P. García-Ruiz, S. M. Olaizola, R. J. Martín-Palma, Surface micro- and nano-texturing of stainless steel by femtosecond laser for the control of cell migration, Sci. Rep. 6 (2016) 36296

${ }^{8}$ C. McDaniel, O. Gladkovskaya, A. Flanagan, Y. Rochev, G. M. O'Connor, In-vitro study on the response of RAW264.7 and MS-5 fibroblast cells on laser-induced periodic surface structures for stainless steel alloys, RSC Adv. 5 (2015) 42548-42558

${ }^{9}$ A. H. A. Lutey, L. Gemini, L. Romoli, G. Lazzini, F. Fuso, M. Faucon, R. Kling, Towards laser-textured antibacterial surfaces, Sci. Rep. 8 (2018) 10112

${ }^{10}$ C. Florian, E. Skoulas, D. Puerto, A. Mimidis, E. Stratakis, J. Solis, J. Siegel, Controlling the wettability of steel surfaces processed with femtosecond laser pulses, ACS Appl. Mater. Interfaces 10 (2018) 36564-36571

${ }^{11}$ J. Bonse, S. Höhm, S. V. Kirner, A. Rosenfeld, J. Krüger, Laser-induced periodic surface structures - A scientific evergreen, IEEE J. Sel. Top. Quant. Electron. 23 (2017) 9000615 ${ }^{12}$ J.-M. Guay, A. C. Lesina, G. Côté, M. Charron, D. Poitras, L. Ramunno, P. Berini, A. Weck, Laser-induced plasmonic colours on metals, Nat. Commun. 8 (2017) 16095

${ }^{13}$ V. Veiko, G. Odintsova, E. Gorbunova, E. Ageev, A. Shimko, Y. Karlagina, Y. Andreeva, Development of complete color palette based on spectrophotometric measurements of steel oxidation results for enhancement of color laser marking technology, Mater. Design 89 (2016) 684-688

${ }^{14}$ S. Gräf, C. Kunz, A. Undisz, R. Wonneberger, M. Rettenmayr, F. A. Müller, Mechanoresponsive colour change of laser-induced periodic surface structures, Appl. Surf. Sci. 471 (2019) 645-651

${ }^{15}$ H. Pauna, X. Shi, M. Huttula, E. Kokkonen, T. Li, Y. Luo, J. Lappalainen, M. Zhang, W. Cao, Evolution of lithium clusters to superatomic $\mathrm{Li}_{3} \mathrm{O}^{+}$, Appl. Phys. Lett. 111 (2017) 103901

${ }^{16}$ Y. Xiong, T. He, Y. Lu, H. Bao, Y. Li, F. Ren, W. Cao, and A. A. Volinsky, Impacts of multiple laser shock processing on microstructure and mechanical property of high-carbon steel, J. Iron Steel Res. Int. 25 (2018) 469-475

${ }^{17}$ V. Veiko, G. Odintsova, E. Ageev, Y. Karlagina, A. Loginov, A. Skuratova, E. Gorbunova, Controlled oxide films formation by nanosecond laser pulses for color marking, Opt. Express 22 (2014) 24342-24347

${ }^{18}$ Y. Lu, X. Shi, Z. Huang, T. Li, M. Zhang, J. Czajkowski, T. Fabritius, M. Huttula, W. Cao, Nanosecond laser coloration on stainless steel surface, Sci. Rep. 7 (2017) 7092

${ }^{19}$ A. Y. Vorobyev, C. Guo, Multifunctional surfaces produced by femtosecond laser pulses, J. Appl. Phys. 117 (2015) 033103

${ }^{20}$ A. Mateo, A. Zapata, G. Fargas, Improvement of mechanical properties on metastable stainless steels by reversion heat treatments, IOP Conf. Series: Materials Science and Engineering 48 (2013) 012001

${ }^{21}$ A. Järvenpää, M. Jaskari, L. P. Karjalainen, Reversed microstructures and tensile properties after various cold rolling reductions in AISI 301LN steel, Metals 8 (2018) 109 
${ }^{22}$ L. V. Taveira, E. Kikuti, N. Bocchi, L. F. Dick, Microcharacterization of colored films formed on AISI 304 by different electrochemical methods, J. Electrochem. Soc. 153 (2006) B411-B416

${ }^{23}$ A. Ben-Yakar, R. L. Byer, Femtosecond laser ablation properties of borosilicate glass, J. Appl. Phys. 96 (2004) 5316

${ }^{24}$ D. Pietroy, Y. D. Maio, B. Moine, E. Baubeau, E. Audouard, Femtosecond laser volume ablation rate and threshold measurements by differential weighing, Opt. Express 20 (2012) 29900-29908

${ }^{25}$ C. A. Schneider, W. S. Rasband, K. W. Eliceiri, NIH image to ImageJ: 25 years of image analysis, Nat. Methods 9 (2012) 671-675

${ }^{26}$ Z. Huang, S .Yang, H. Zhang, M. Zhang, W. Cao, Replication of leaf surface structures for light harvesting, Sci. Rep. 5 (2015) 14281

${ }^{27}$ Z. Huang, C. Cai, L. Kuai, T. Li, M. Huttula, W. Cao, Leaf-structure patterning for antireflective and self-cleaning surfaces on Si-based solar cells, Solar Energy 159 (2018) $733-741$

${ }^{28}$ M. Rohloff, S. K. Das, S. Höhm, R. Grunwald, A. Rosenfeld, J. Krüger, J. Bonse, Formation of laser-induced periodic surface structures on fused silica upon multiple crosspolarized double-femtosecond-laser-pulse irradiation sequences, J. Appl. Phys. 110 (2011) 014910

${ }^{29}$ R. W. G. Hunt, The Reproduction of Colour. (Wiley-IS\&T Series in Imaging Science and Technology, 2004)

${ }^{30}$ H. Y. Zheng, G. C. Lim, X. C. Wang, J. L. Tan, J. Hilfiker, Process study for laserinduced surface coloration, J. Laser Appl. 14 (2002) 215-220

${ }^{31}$ F. Luo, W. Ong, Y. Guan, F. Li, S. Sun, G. C. Lim, M. Hong, Study of micro/nanostructures formed by a nanosecond laser in gaseous environments for stainless steel surface coloring, Appl. Surf. Sci. 328 (2015) 405-409

${ }^{32}$ C. Noguez, Surface plasmons on metal nanoparticles: the influence of shape and physical environment, J. Phys. Chem. C 111 (2007) 3806-3819

${ }^{33}$ A. P. Grosvenor, B. A. Kobe, M. C. Biesinger, N. S. McIntyre, Investigation of multiplet splitting of Fe 2p XPS spectra and bonding in iron compounds, Surf. Interface Anal. 36 (2004) 1564-1574

${ }^{34}$ C. Biagioni, P. Marco, The systematics of the spinel-type minerals: an overview, Am. Mineral. 99 (2014) 1254-1264

${ }^{35}$ A. M. Huntz, A. Reckmann, C. Haut, C. Sévérac, M. Herbst, F. C. T. Resende, A. C. S. Sabionic, Oxidation of AISI 304 and AISI 439 stainless steels, Mater. Sci. Eng. A 447 (2007) 266-276

${ }^{36}$ A. M. P. Simões, M. G. S. Ferreira, B. Rondot, M. da Cunha Belo, Study of passive films formed on AISI 304 stainless steel by impedance measurements and photoelectrochemistry, J. Electrochem. Soc. 137 (1990) 82-87

${ }^{37}$ J. Torrent, V.Barrón, Diffuse reflectance spectroscopy of iron oxides, Encyclopedia of Surface and Colloid Science 1 (2002) 1438-1446 
${ }^{38}$ V. D’Ippolito, G. B. Andreozzi, U. Hålenius, H. Skogby, K. Hametner, D. Günther, Color mechanisms in spinel: cobalt and iron interplay for the blue color, Phys. Chem. Miner. 42 (2015) 431-439

${ }^{39}$ L. T.-T. Huong, T. Häger, W. Hofmeister, C. Hauzenberger, D. Schwarz, P. V. Long, U. Wehmeister, N. N. Khoi, N. T. Nhung, Gemstones from Vietnam: an update, Gems Gemol. 48 (2012) 158-176

${ }^{40}$ B. Smits, An RGB to spectrum conversion for reflectances, J. Graphics Tools 4 (1999) $11-22$

${ }^{41}$ S. A. Burns, Generating reflectance curves from sRGB triplets, arXiv:1710.05732 (2017) 